\title{
Optic flow is calibrated to walking effort
}

\author{
Jonathan R. Zadra ${ }^{1}$ - Dennis R. Proffitt ${ }^{2}$
}

Published online: 24 March 2016

(C) Psychonomic Society, Inc. 2016

\begin{abstract}
Through experience, people learn that a given magnitude of walking produces an associated magnitude of optic flow. Artificially altering this relationship has both behavioral and perceptual consequences: walking on a treadmill results in zero translational optic flow and causes people to subsequently drift forward when attempting to walk in place while blindfolded (they have learned that forward walking is required to remain stationary). Similarly, after walking on a treadmill people perceive the walking distance to targets to be greater (they have recalibrated the magnitude of walking required to reach the target). While the measurement unit for walking magnitude in this relationship has been treated as walking speed (stride length $*$ [steps / time]), recent experiments suggest that walkable distances may instead be measured in bioenergetic units (i.e., the magnitude of energy required to produce a given magnitude of optic flow). In the first experiment, zero translational optic flow was paired with a constant walking speed, and walking energy was manipulated by varying the incline of the treadmill. Participants who walked on an inclined treadmill drifted farther while attempting to walk in place than participants who walked on a flat treadmill. A follow-up experiment presented optic flow via an immersive virtual environment, and no difference between flat and inclined treadmill walking was found, thereby
\end{abstract}

Jonathan R. Zadra

j.zadra@utah.edu

1 Department of Psychology, University of Utah, Salt Lake City, UT, USA

2 Department of Psychology, University of Virginia, Charlottesville, VA, USA showing that the effect found in the first experiment was not an artifact of biomechanical differences associated with flat versus inclined treadmill walking. The results support the hypothesis that walking magnitude is scaled by bioenergetic units.

Keywords Embodied cognition · Perceptual learning · Physiological psychology $\cdot$ Visual $\cdot$ Perception

The current study investigated the visual-motor recalibration process responsible for updating the associative relationship between walking and optic flow. Walking produces optic flow, which consists of environment-specifying changes in visual angles. Moreover, as people walk they discover the relationship between the magnitudes of optic flow that are produced by varying magnitudes of walking. The current study investigated the effective variables specifying walking magnitude. More precisely, our research question in this context of visual-motor recalibration was: What is the measurement unit for walking magnitude?

A number of studies have used a treadmill to investigate the relationship between walking and the visual information that accompanies it. Normally, optic flow is coupled to walking, such that walking at $3 \mathrm{mph}$ produces $3 \mathrm{mph}$ of optic flow. On a treadmill, regardless of walking or running speed, there is no translational optic flow. A number of experiments have shown that decoupling rate of walking from rate of optic flow has perceptual and behavioral effects, and anyone who has walked or run on a treadmill has experienced one of these: upon dismounting the treadmill one gets the strange feeling for a short while of moving too quickly when walking (Pelah \& Barlow, 1996).

The effect of treadmill walking on motor behavior was first reported by Anstis (1995) who showed that, after running 
forwards or backwards on a treadmill, blindfolded participants attempting to jog in place drifted either forwards or backwards, respectively. These findings have been extended in subsequent studies (cf., Durgin \& Pelah, 1999). Anstis' assessment of fore/aft drift-for blindfolded participants attempting to walk or jog in place - is the dependent measure used in the current studies.

In related work, Gordon, Fletcher, Melvill Jones, \& Block (1995) had participants walk on the outside edge of a large, rotating disk so as to remain stationary relative to the room for $2 \mathrm{~h}$. The disk was masked from view, but otherwise they were able to see freely. Next they were asked to march in place while blindfolded. Participants tended to drifted forward along a curved trajectory. The results demonstrate that, when there was a mismatch between walking trajectory (curved, which normally would produce a pattern of optic flow associated with a curvilinear trajectory) and optic flow (in this case, zero forward optic flow and zero rotational optic flow), participants recalibrated such that that turning in the direction of the circle while walking forward is required to remain in place facing straight ahead.

In addition to recalibration studies showing a drifting effect for participants attempting to walk in place, it has been demonstrated that recalibration likewise affects walking to targets. Rieser, Pick, Ashmead, and Garing (1995) had participants walk on a treadmill as it was pulled on a trailer behind a tractor, thereby allowing the experimenters to independently adjust both the walking speed and the speed of optic flow. Next, participants viewed a series of targets. After viewing each target, they were blindfolded and asked to walk to the targets. Participants whose walking rate on the treadmill was greater than the optic flow rate (i.e., the speed of the tractor) overshot the targets, whereas participants whose walking rate was less than the optic flow rate undershot the targets. In the first case, the visual-motor system had been recalibrated to anticipate that more walking was required to traverse a distance, while in the second case, it had recalibrated to anticipate that less walking was required. Similarly, Proffitt, Stefanucci, Banton, and Epstein (2003) used virtual reality (VR) to present participants walking on a treadmill with visual information of a stationary world or of a world that was appropriately moving at their walking rate. Participants marched in place while blindfolded before and after walking on the treadmill, and only those who experienced zero flow drifted forward more on the second occasion than the first. (In these and other studies, a slight tendency to drift forward on all occasions was found, and slightly more so following treadmill walking.) The participants in the zero flow condition had recalibrated and, thereby, implicitly expected that forward walking was required to remain stationary. Estimates of the distance to targets were similarly affected: participants' estimates of the distance to targets were greater following a period of walking on a treadmill with zero optic flow relative to their pre-walking estimates (Proffitt et al., 2003).
While there is evidence that recalibration occurs when there are changes in the relationship between the speed specified by optic flow and walking speed, the effective variable related to walking speed has not been determined. Walking speed can be thought of as stride length multiplied by steps per second, or as a bioenergetic variable such as calories per second. Consider the study by Rieser et al. (1995), in which some participants walk faster in some conditions than others. Walking faster would seem to require more energy and thus provide one potential way to differentiate between speed versus energy being calibrated to optic flow. However, there is a linear relationship between rate of energy expenditure and walking speed: while faster walking or running speeds require more energy per unit of time, they actually require about the same amount of energy per unit of distance (McArdle, Katch, \& Katch, 2010), leaving the ratio of energy to optic flow unchanged for canonical optic flow. Note that we do not get an aftereffect when we transition from running to walking in everyday situations.

A recent study supports the hypothesis that energy, rather than speed, is what is most important. Using a treadmill and a virtual environment, White, Shockley, and Riley (2013) created situations with altered relationships between energy expenditure and the optically specified distance that was traversed (individually changing either the walking rate, grade of inclination, or optic flow rate while keeping the other two variables constant). They found that these manipulations influenced subsequent attempts to traverse what was perceived to be the same distance. Increasing energy expenditure while keeping the optically specified distance constant, for example, caused participants to traverse a greater distance. While this study shows that the perception of distance is scaled by energy, its research focus was not on the process of visual-motor recalibration.

Given evidence that walkable extents are scaled by the bioenergetic costs of walking (Zadra, Weltman, \& Proffitt, 2015; White, et al., 2013), walking energy rather than walking speed may also be the effective variable in the associative relationship between walking magnitude and optical flow magnitude. To test this hypothesis, we used a treadmill and virtual environment (Experiment 2) to manipulate the three relevant variables: (1) walking speed, (2) walking effort, and (3) optical flow speed (see Fig. 1). Following treadmill walking, the assessed dependent variable was magnitude of forward drift for blindfolded participants attempting to walk in place. In Experiment 1, both walking speed and walking effort were mismatched with the resultant zero optic flow, whereas in Experiment 2, both walking speed and effort resulted in appropriate optic flow.

In both conditions of Experiment 1, translational optic flow was zero and walking speed was $3 \mathrm{mph}$; thus, if the effective variable for recalibration is walking speed, then forward drift should be equivalent across conditions. In this experiment, 


\begin{tabular}{|c|c|c|c|c|c|}
\hline & Condition & Walking Speed & Walking Effort & $\begin{array}{c}\text { Apparent } \\
\text { Optic Flow }\end{array}$ & $\begin{array}{l}\text { Recalibration } \\
\text { Required? }\end{array}$ \\
\hline \multirow{2}{*}{ Experiment 1} & & $3 \mathrm{mph}$ & E & $0 \mathrm{mph}$ & Yes \\
\hline & & $3 \mathrm{mph}$ & $E+\delta$ & $0 \mathrm{mph}$ & Yes \\
\hline \multirow{2}{*}{ Experiment 2} & & $3 \mathrm{mph}$ & E & $3 \mathrm{mph}^{*}$ & No \\
\hline & & $3 \mathrm{mph}$ & $E+\delta$ & $3 \mathrm{mph}^{*}$ & No \\
\hline
\end{tabular}

Fig. 1 In Experiment 1, quantities of walking speed and walking effort were paired with zero translational optic flow. Recalibration is required because these magnitudes of both walking speed and walking effort have been previously paired in experience with resultant optic flow. In Experiment 2, walking speed and walking effort were paired with proper optic flow. Walking speed was the same in each condition and resulted in the appropriate optic flow. Energy (E) varied across conditions due to the incline of the treadmill. In both cases of treadmill incline, the perceived optic flow was appropriate for the amount of energy expenditure $(\mathrm{E}$ or $\mathrm{E}+\delta)$ given past experience walking on flat ground or inclines. *Actual optical flow was $4.7 \mathrm{mph}$. See text.

diabetes or cardiac problems. Three participants were excluded for procedural errors: one was run with the lights off while on the treadmill, one was unable to walk at the defined treadmill speed, and one's drift measurements were taken incorrectly.

\section{Apparatus}

An exercise treadmill (Precor 9.1; http://www.precor.com) set at either a flat, $0^{\circ}$ incline or a $10^{\circ}$ incline was used to simulate locomotion.

\section{Procedure}

The treadmill was set to the appropriate orientation before participants arrived. Upon arrival at the laboratory, participants were given a general description of the experiment and provided informed consent.

Because participants would be led between the marching location and the treadmill while blindfolded, participants were first led through the transfer procedure with full vision so that they would know what to expect. The experimenter informed the participants that they would be marching in place for a short time, and demonstrated how they should march: a slow, steady rhythm (at about 100 steps per minute) with knees brought high, almost to waist height. The experimenter then asked the participants to demonstrate marching in place, and instructed them in any necessary corrections until the style was correct. 
Even with appropriate optic flow, there is a tendency to drift forward slightly when blindfolded participants attempt to march in place (Anstis, 1995; Durgin \& Pelah, 1999; Gordon et al., 1995; Proffitt et al., 2003). For this reason, a pre-manipulation measure of forward drift while attempting to walk in place was recorded so that baseline drift could be accounted for in the analyses. Participants were blindfolded and led to an open area in the room. Their location was surreptitiously marked on the floor, and they were instructed to begin marching in place. After exactly $20 \mathrm{~s}$, they were instructed to stop and stand still, and their new location was again marked. Participants were then led to the treadmill and instructed to hold on to a crossbar in front of them. The treadmill was slowly accelerated to $3 \mathrm{mph}$, at which point a timer was started and participants were instructed to remove the blindfold. After $10 \mathrm{~min}$, participants donned the blindfold again, and the treadmill was slowed to a stop. They were led back to an open area of the room, their location was marked, and they were instructed to begin marching. They were told to stop after exactly $20 \mathrm{~s}$, and their location was marked again. Participants were guided to a new location to prevent feedback on their performance, and then allowed to remove the blindfold.

Participants were debriefed, and then the straight-line distance between the starting and ending locations for each marching occasion were measured (some participants followed a slightly curved path, but the primary direction of motion was forward on all occasions).

\section{Results and discussion}

A linear mixed-effects model was used to investigate the possible predictors of marching drift. One variable each was dummy coded for occasion (pre/post) and treadmill incline (flat/ incline), and drift distance was predicted from the additive effects of these terms and an interaction term between occasion and incline, with a subject-level random intercept. The analysis revealed statistically significant coefficients for occasion, and the interaction between occasion and incline in predicting the drift distance (meters). As expected, all participants drifted further after walking on the treadmill than they had before $(\beta=0.55, P<.001)$, and participants who walked on the inclined treadmill drifted farther on the second occasion than those who walked on the flat treadmill $(\beta=0.31, P=$ 0.018 ; see Fig. 2). There was no significant difference in pretreadmill drift distances between incline conditions $(\beta=$ $<0.00, P=0.996$ ).

The results support the hypothesis that the calibration between walking and optic flow is not (or not entirely) based on walking speed, but rather on the energy required to walk. In both conditions, participants walked at the same speed, but participants who walked on the inclined treadmill had to expend more energy to walk at that speed.

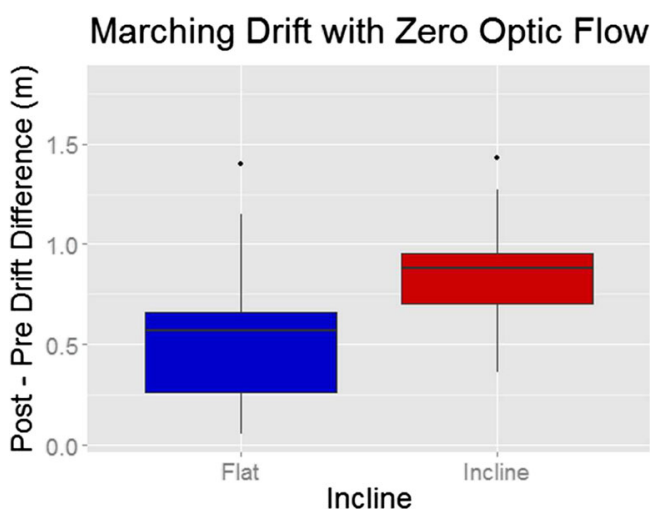

Fig. 2 Box-and-whisker plot of post-/pre-drift difference grouped by condition (whiskers indicate variability outside the 1 st and 3rd quartiles)

Despite strong supporting results, there are potentially other factors besides the energy required to walk that were not matched between the conditions. For instance, the dynamics of walking up an incline could perhaps have worked different muscles, and resulted in different marching-in-place dynamics that produced greater drift. In order to control for this and other potential differences between flat and incline walking, the follow-up study replicated all aspects of Experiment 1 but removed the need for recalibration: all participants received canonical optic flow through a VR display.

\section{Experiment 2: Treadmill incline with canonical optic flow}

\section{Method}

\section{Participants}

Forty-six volunteers from the University of Virginia community (28 women; mean age $=18.98$ years, $\mathrm{SD}=1.03$ years $)$ received credit for participating. Participants were required to have normal or corrected-to-normal vision, speak English as their first language, and have no history of diabetes or cardiac problems. Six participants were excluded: three because the treadmill safety was tripped causing it to stop prematurely, one because the VR headset could not be properly fitted, and one because they drifted so far to one side that they bumped into an object and stopped (the last was in the flat condition).

\section{Apparatus}

\section{Treadmill}

As in Experiment 1, an exercise treadmill (Precor 9.1) set at either a flat, $0^{\circ}$ incline or a $10^{\circ}$ incline was used to simulate locomotion. 
Fig. 3 The virtual environment (golf cart was not shown in the present study)

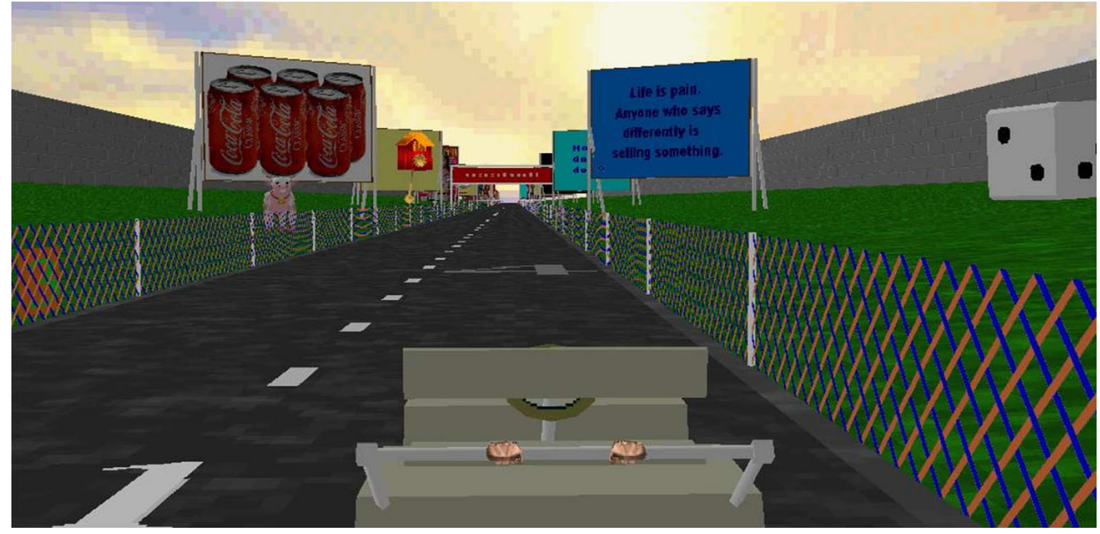

\section{Virtual reality}

To simulate optic flow, a virtual environment (VE) was viewed through a head-mounted display (Virtual Research V8 HMD) created and rendered with Alice99. Using a Dell Precision 360 computer equipped with a GeForce 4 MX420 and GeForce 4 MX200, the HMD displayed stereo images at 640 by 480 at 60 frames per second with a horizontal field of view of $48^{\circ}$. Head movements were registered to update the VE seen through the HMD using an Intersense IS-900 motion tracking system.

\section{Stimuli}

The VE consisted of a highway with billboards and various landmarks along the sides (see Fig. 3). The VE was the same as that used in a previous treadmill study that investigated the perception of walking speed in a virtual environment (for specific details on the VE, see Banton, Stefanucci, Durgin, Fass, \& Proffitt, 2005; newer hardware was used, and the golf cart was not shown). As participants walked on the treadmill, the virtual camera moved through the VE at a speed that had been determined previously to match perceived speed to the treadmill speed. ${ }^{1}$ The orientation of the camera matched the participants' direction of gaze as determined by the head tracker, and participants were encouraged to look around freely.

\section{Procedure}

The procedure was identical to that of Experiment 1, except that, after getting on the treadmill, participants closed their eyes, removed the blindfold, and put on the HMD before reopening their eyes (participants were also practiced in

\footnotetext{
${ }^{1}$ Banton et al., (2005) determined that with an HMD, straight-ahead optic flow is perceived to be slower than an equivalent walking speed on the treadmill; a camera speed of $4.7 \mathrm{mph}$ was used in the current experiment because it best matched a treadmill walking speed of $3 \mathrm{mph}$.
}

donning the HMD from the initial training phase of the experiment). After walking for $10 \mathrm{~min}$, the treadmill was stopped, and the participants quickly closed their eyes, removed the HMD, and replaced the blindfold, and then continued with the second marching procedure as in Experiment 1.

\section{Results and discussion}

Analyses proceeded identically to that of Experiment 1. A linear mixed-effects model was used to investigate the possible predictors of marching drift. One variable each was dummy coded for occasion (pre/post) and treadmill incline (flat/ incline), and drift distance was predicted from the additive effects of these terms and an interaction term between occasion and incline, with a subject-level random intercept. The analysis revealed a statistically significant coefficient for occasion, but none for any coefficient that included the incline condition. As expected, all participants drifted slightly further after walking on the treadmill than they had before $(\beta=0.14$, $P<.001$ ), but unlike Experiment 1, participants who walked on the inclined treadmill did not drift farther on the second occasion than those who walked on the flat treadmill $(\beta=$ $<0.00, P=0.92$, see Fig. 4).

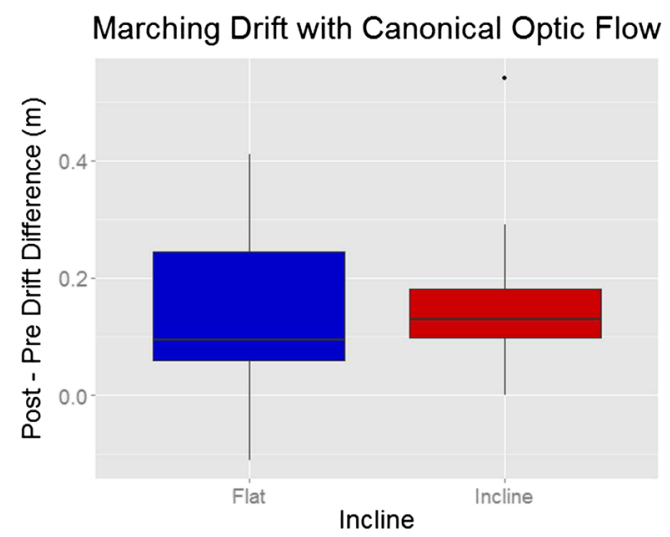

Fig. 4 Box-and-whisker plot of post-/pre-drift difference grouped by condition (whiskers indicate variability outside the 1st and 3rd quartiles) 
The expected null results provide evidence that the results of Experiment 1 were not artifactually due to unanticipated differences between the conditions such as differing biomechanics of walking on an inclined versus a flat treadmill. While all participants drifted slightly more after walking on the treadmill, the amount was less than one-third of that which occurred without optic flow, and, as discussed previously, some small amount of drift is expected based on previous experiments.

Walking in place with zero optic flow (Experiment 1) produced a mismatch between both walking speed and effort and resultant optic flow that necessitated recalibration. If the relevant mismatch was only between walking speed and optic flow, then there should have been no difference between treadmill conditions. If the calibration depends on the energy required to walk, however, then the inclined treadmill condition should induce a greater after effect than the flat treadmill condition. This latter result was found. In Experiment 2, there was no mismatch between either walking speed or effort and resultant optic flow rate. Consequently, no after effect was observed in either condition.

\section{Conclusion}

As hypothesized, visual-motor calibration between walking and optic flow is dependent on the energy required to walk, rather than (or to a greater extent than) the speed of walking. Maintaining the same walking speed under conditions of zero translational optic flow while manipulating the energy required to walk caused a visual-motor recalibration that differed depending on the amount of energy expenditure. When optic flow was appropriate for both walking speed and effort, no visual-motor recalibration was necessary, and the energy manipulation had no effect.

These results support the idea that the perception-action system is sensitive to bioenergetic costs and bioenergetic state. They also indicate that the relationship between perception and action can be learned (and quite rapidly), thus providing an answer to the question of how the scale of walkable distances can be established via visually guided walking. Simultaneously experiencing the bioenergetic consequences of walking and the resultant optic flow allows the relationship between the two to be established, and that relationship can serve as the scaling unit for walkable distances.

Author's note The reported research comprised a part of the primary author's doctoral dissertation at the University of Virginia. Committee members were Dennis Proffitt (chair), Gerald Clore, Michael Kubovy, and Arthur Weltman. Jonathan Zadra is now in the Psychology Department at the University of Utah.

\section{References}

Anstis, S. (1995). After effects from jogging. Experimental Brain Research, 103(3), 476-478.

Banton, T., Stefanucci, J. K., Durgin, F. H., Fass, A., \& Proffitt, D. R. (2005). The perception of walking speed in a virtual environment. Presence: Teleoperators and Virtual Environments, 14(4), 394-406. doi:10.1162/105474605774785262

Durgin, F. H., \& Pelah, A. (1999). Visuomotor adaptation without vision? Experimental Brain Research, 127(1), 12-18. doi:10.1007/ s002210050769

Gordon, C. R., Fletcher, W. A., Melvill Jones, G., \& Block, E. W. (1995). Adaptive plasticity in the control of locomotor trajectory. Experimental Brain Research Experimentelle Hirnforschung Experimentation Cerebrale, 102(3), 540-545. doi:10.1007/ BF00230658

McArdle, W. D., Katch, F. I., \& Katch, V. L. (2010). Exercise Physiology: Energy, Nutrition, and Human Performance (7th ed.). Baltimore: Lippincott, Williams \& Wilkins.

Pelah, A., \& Barlow, H. B. (1996). Visual illusion from running. Nature, 381(6580), 283. doi:10.1038/381283a0

Proffitt, D. R., Stefanucci, J. K., Banton, T., \& Epstein, W. (2003). The role of effort in perceiving distance. Psychological Science, 14(2), $106-112$.

Rieser, J. J., Pick, H. L., Ashmead, D. H., \& Garing, A. E. (1995). Calibration of human locomotion and models of perceptual-motor organization. Journal of Experimental Psychology: Human Perception and Performance, 21(3), 480-497. doi:10.1037/00961523.21.3.480

White, E., Shockley, K., \& Riley, M. A. (2013). Multimodally specified energy expenditure and action-based distance judgments. Psychonomic Bulletin \& Review, 20(6), 1371-1377. doi:10.3758/ s13423-013-0462-8

Zadra, J. R., Weltman, A. L., \& Proffitt, D. R. (2015). Walkable distances are bioenergetically scaled. Journal of Experimental Psychology: Human Perception and Performance, 42(1), 39-51. doi:10.1037/ xhp0000107 